Supporting Information

\title{
Superelastic Triple-Network Polyorganosiloxane-Based Aerogels as Transparent Thermal Superinsulators and Efficient Separators
}

Guoqing Zu, ${ }^{1, *}$ Kazuyoshi Kanamori, ${ }^{2}$ Xiaodong Wang, ${ }^{1, *}$ Kazuki Nakanishi,${ }^{3}$ Jun Shen ${ }^{1}$

${ }^{1}$ School of Materials Science and Engineering, Shanghai Key Laboratory of Special Artificial Microstructure Materials and Technology, School of Physics Science and Engineering, Tongji University, Shanghai 201804, P. R. China

${ }^{2}$ Department of Chemistry, Graduate School of Science, Kyoto University, Kitashirakawa, Sakyo-ku, Kyoto 606-8502, Japan

${ }^{3}$ Institute of Materials and Systems for Sustainability, Nagoya University, Furo-cho, Chikusaku, Nagoya 464-8601, Japan

*Corresponding authors :

E-mail: guoqingzu@tongji.edu.cn (G. Zu)

xiaodong_wang@tongji.edu.cn (X.Wang)

This PDF file includes:

Figures S1 to S6

Table S1 
Figures

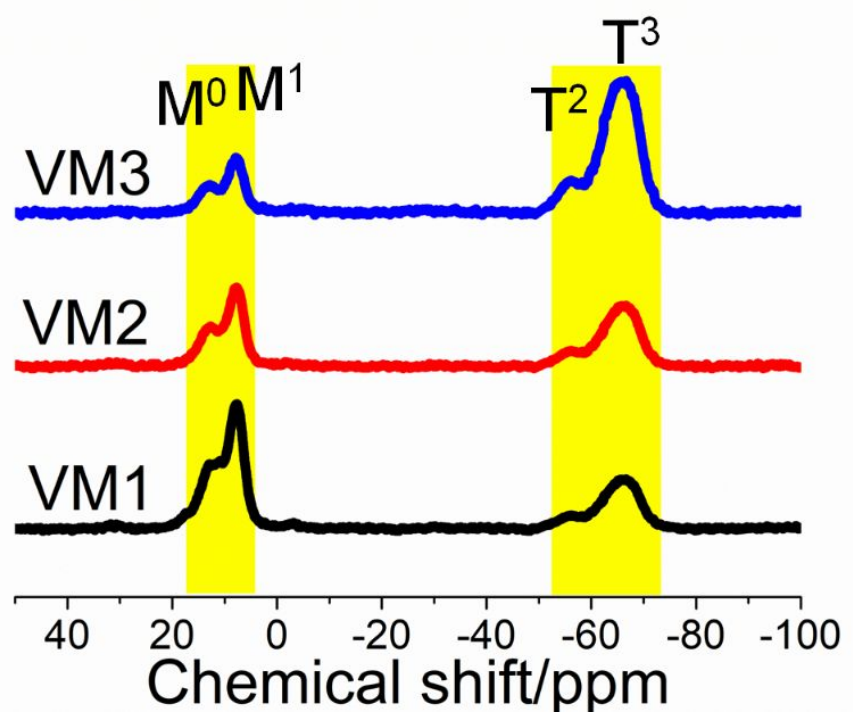

Figure S1. ${ }^{29} \mathrm{Si}$ CP/MAS NMR spectra of typical polyorganosiloxane-based aerogels.

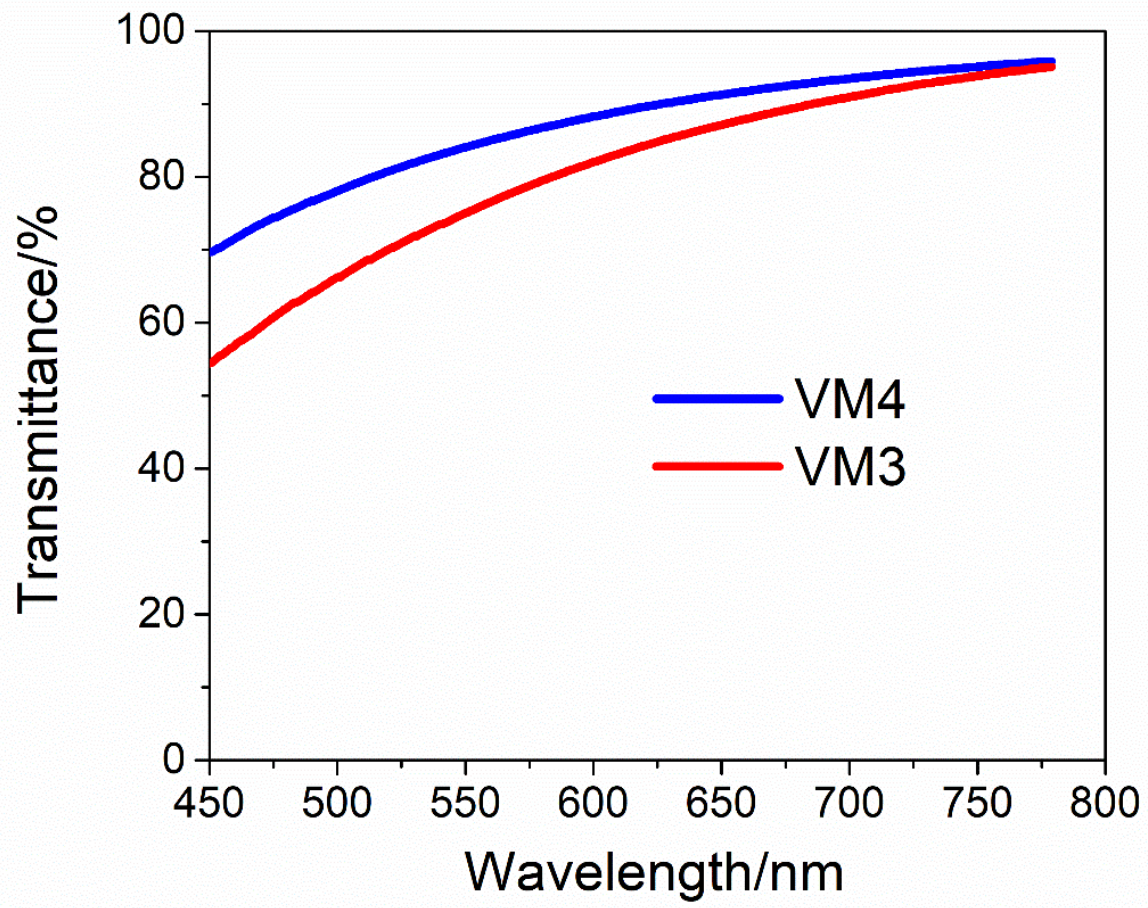

Figure S2. Light transmittance spectrum of $2 \mathrm{~mm}$ thick aerogels VM3 and VM4. 

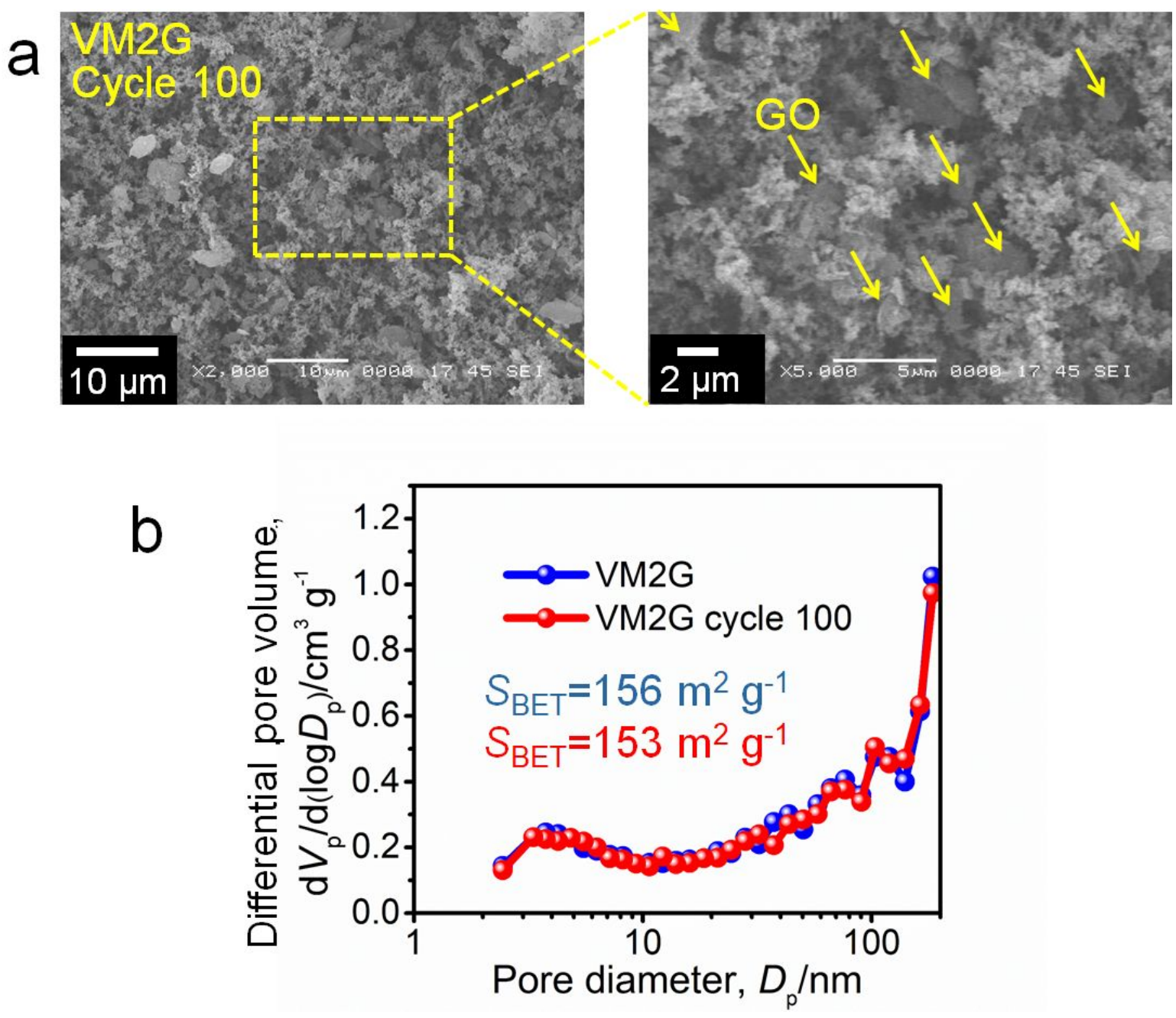

Figure S3. (a) Morphology and (b) pore size distribution of VM2G after compressiondecompression with $80 \%$ strain for 100 cycles. 

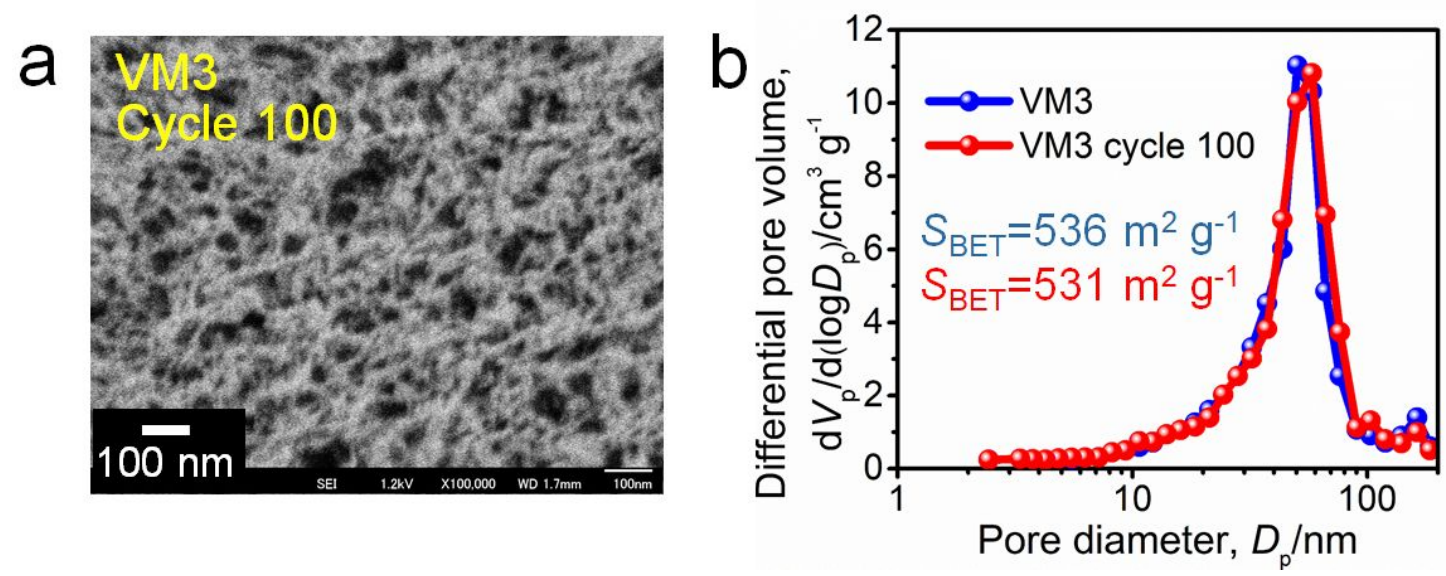

Figure S4. (a) Morphology and (b) pore size distribution of VM3 after compressiondecompression with $80 \%$ strain for 100 cycles.
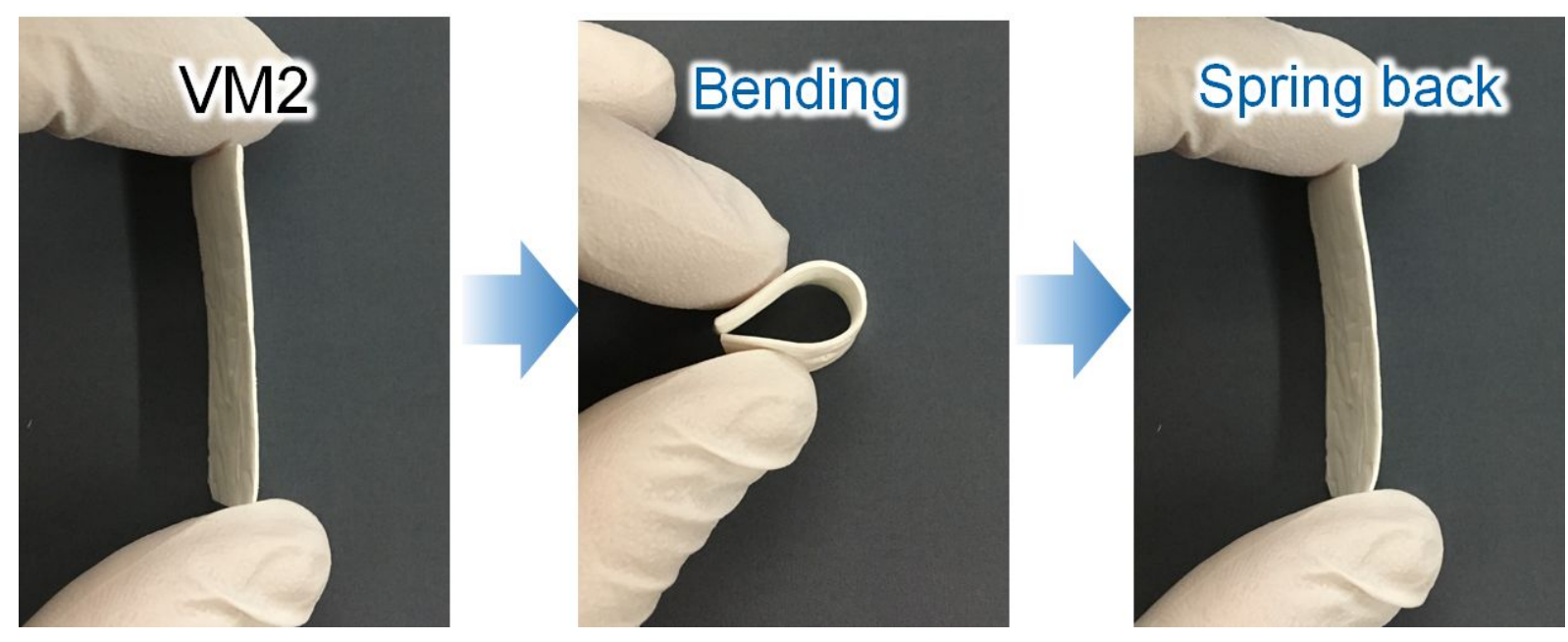

Figure S5. Photographs of a hand bending test on VM2, showing high bending flexibility. 


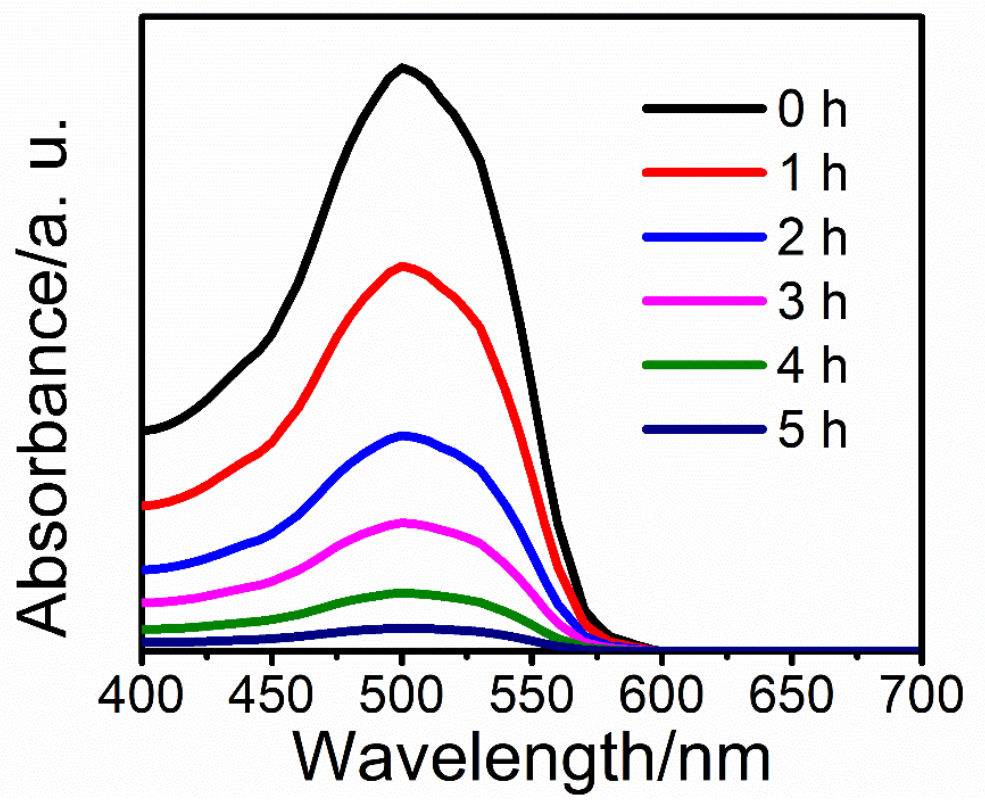

Figure S6. Absorption spectra of sudan III solution in the presence of VM2G.

\section{Table:}

Table S1. The atomic percentages of $\mathrm{Si}, \mathrm{C}$, and $\mathrm{O}$ in typical polyorganosiloxane-based aerogels obtained from XPS data.

\begin{tabular}{cccc}
\hline Sample & $\mathrm{Si} / \%$ & $\mathrm{C} / \%$ & $\mathrm{O} / \%$ \\
\hline VM3 & 25.32 & 41.05 & 33.63 \\
VM2 & 21.50 & 53.86 & 24.64 \\
VM2G & 20.13 & 56.89 & 22.98 \\
\hline
\end{tabular}

